\title{
Extra-intestinal manifestations in inflammatory bowel disease: Etiopathogenesis and management
}

\author{
Clara Ramos-Belinchón, Luis Menchén, and Ignacio Marín-Jiménez* \\ Department of Gastroenterology, Hospital General Universitario Gregorio Marañón, Madrid, Spain
}

\begin{abstract}
Inflammatory bowel disease (IBD) can be considered a systemic inflammatory disease since it can present with a variety of extra-intestinal manifestations (EIMs). Arthropathies are the most common manifestation, but EIMs can occur in a wide variety of organs. The relevance of EIMs lies in its impact on quality of life. Even some EIMs may be associated with a decreased survival as occurs in primary sclerosing cholangitis (PSC), which is related to an increased risk of cholangiocarcinoma. The pathogen of EIMs is still partially unknown and treatments are not always effective. Some EIMs follow the course of the intestinal activity and may be treated as an intestinal flare. Others have an independent course and are difficult to treat. In this article, we review the pathogenesis of EIMs, their clinical manifestations, and the available treatments.
\end{abstract}

Keywords: Inflammatory bowel disease. Extra-intestinal manifestation. Crohn's disease. Ulcerative colitis.

\section{Introduction}

Crohn's disease (CD) and ulcerative colitis (UC) are systemic inflammatory diseases that mainly affect the gastrointestinal tract but can manifest with extra-intestinal symptoms in up to $50 \%$ of patients ${ }^{1}$. These extraintestinal manifestations (EIMs) have a significant impact on the quality of life of patients and, in the case of primary sclerosing cholangitis (PSC), on survival due to an increased risk of neoplasia ${ }^{2}$. EIM can be diagnosed before, after, or simultaneously with inflammatory bowel disease (IBD). There are even some very specific EIMs (like PSC) that should make us actively search for an underlying IBD'. EIMs affect multiple organs (such as skin, eyes, muscles, joints, liver, and bile duct) and can run a parallel or independent course from intestinal activity ${ }^{3,4}$.

In this article, we review the epidemiology and pathogen of EIMs and describe the most frequent EIMs as well as their current management.

\section{Epidemiology}

Between 6 and $47 \%$ of patients with IBD develop at least one EIM throughout their lives, with arthralgia/ arthritis being the most frequent EIM $^{5-13}$. The prevalence of the most frequent EIMs is described in table 1. It is more common in patients with CD than with UC and affects more women ${ }^{13-15}$.

The likelihood of developing EIMs increases with the duration of bowel disease and is higher in patients who already have another EIM $^{13}$. In most patients, EIMs occur after IBD diagnosis, but about $25 \%$ of cases appear before it ${ }^{1}$.

\section{Pathogenesis}

The pathogenesis of EIMs is still partially unknown. There are two theories that explain the development of these manifestations. The first describes the occurrence of extraintestinal symptoms as the extension of a specific immune response to other systems. The 
Table 1. Prevalence of the most frequent EIMs $40,41,51,53$

\begin{tabular}{|l|c|}
\hline Axial arthropathy & Up to $25 \%$ \\
\hline Peripheral arthropathy & $5-20 \%$ \\
\hline Erythema nodosum & Up to $15 \%$ \\
\hline Pyoderma gangrenosum & $0,4-2 \%$ \\
\hline Episcleritis & $2-5 \%$ \\
\hline Anterior uveitis & $0,5-3 \%$ \\
\hline Primary sclerosing cholangitis & $4-5 \%$ \\
\hline Osteoporosis & $4-9 \%$ \\
\hline
\end{tabular}

second argues that EIMs are independent inflammatory processes but that appear as a consequence of IBD or by shared environmental or genetic factors. Most likely, several pathogenic mechanisms are involved in the development of EIMs ${ }^{16}$.

\section{Extension of specific immune response from the intestine}

It has been demonstrated an ectopic expression of cytokines and adhesion molecules that in healthy patients are limited to the intestine. Specifically, the ectopic expression of MAdCAM-1 and CCL25 has been identified in the vascular endothelium of the portal tract of patients with PSC associated with IBD ${ }^{17,18}$.

Another pathogenic mechanism in this sense is the increase in chemokines and adhesion molecules non-specific of the gastrointestinal tract that can lead to the recruitment of intestinal leukocytes at an extra-intestinal site ${ }^{16,19}$.

There could be an immune reaction on other systems mediated by circulating antibodies and immune complexes. Shared epitopes of the colon with other organs (eyes, joints, skin, and biliary epithelium) have been found ${ }^{20-22}$. However, this causal mechanism in the development of EIMs has not been demonstrated yet.

Finally, the translocation of microbial antigens derived from the intestinal microbiota could be another pathogenic mechanism. Patients with IBD-associated PSC have a different gut microbiota and the transport of microbial antigens into the portal circulation could activate an immune response through $\alpha 4 \beta 7$-MAdCAM-123,24.

\section{Independent inflammatory events}

Patients with IBD have increased mucosal and systemic levels of pro-inflammatory mediators such as interleukin (IL)-6, tumor necrosis factor (TNF- $\alpha$ ), interferon, and vascular endothelial growth factor, as well as bacterial lipopolysaccharide (LPS) that can promote immune activation in other systems ${ }^{25}$. The innate immune system is also involved through a phenomenon called "neutrophil priming"26, which means that neutrophils present a greater response when activated and produce a higher amount of IL-1 $\beta$ and TNF- $\alpha$. In addition, there could be an alteration in hematopoiesis ${ }^{27}$.

The intestinal microbiota seems to be related to the development of EIMs. Some of the mechanisms that could be involved in this would be translocation of bacteria or microbial products such as LPS due to an alteration of the intestinal barrier, and the production of metabolites derived from the microbiota that stimulate the immune system ${ }^{28-30}$.

\section{Musculoskeletal manifestations}

Arthropathies associated with IBD are the most frequent EIM and are included in the group of spondyloarthropathies. They are divided into axial and peripheral. They must be differentiated from arthralgias, which are a very common symptom in patients with IBD. In addition to these two forms that will be explained below, patients with IBD may have drug-induced joint symptoms. Treatment with corticosteroids increases the risk of osteoporosis and avascular bone necrosis, and anti-TNF treatment is associated with a lupus-like phenomenon ${ }^{2}$.

\section{Axial arthropathy}

Axial arthropathy is usually independent of IBD. Axial involvement is characterized by sacroilitis that can be accompanied by vertebral involvement and includes a spectrum of entities from non-radiological axial spondyloarthropathy to ankylosing spondylitis (Fig. 1). Its prevalence ranges between $12 \%$ and $46 \%$ of patients with IBD and an association with HLA-B27 has been demonstrated, especially in patients with ankylosing spondylitis ${ }^{31-33}$.

\section{Peripheral arthropathy}

Peripheral arthropathy has been classically classified into pauciarticular or type 1 and polyarticular or type 2 . Type 1 affects fewer than 5 joints and usually manifests in large joints, especially knees. The course of this type of arthropathy runs in parallel with intestinal activity and is usually self-limited, with a duration of $<10$ weeks. Type 2 affects five or more small joints, the metacarpophalangeal joints being the most common. The course of type 2 arthropathy is independent of IBD activity, and symptoms tend to last longer than in type $1^{10,34}$. 


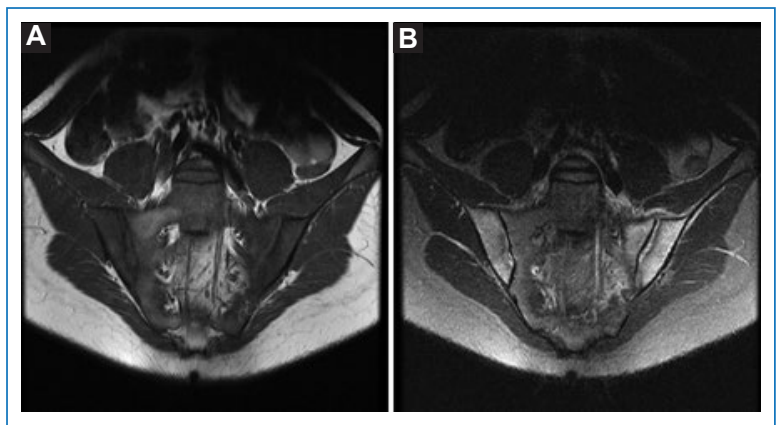

Figure 1. Magnetic resonance imaging of the sacroiliac joints of a 17-year-old patient with Crohn's disease and with back and left sacroiliac pain. A: sequences T1-weighted images and B: STIR shows bilateral sacroiliac osteitis.

\section{Treatment}

The European consensus document of the European Crohn's and Colitis Organization (ECCO) ${ }^{2}$ recommends for the treatment of axial arthropathy intensive physical therapy and short-term nonsteroidal anti-inflammatory drugs (NSAIDs). They do not recommend keeping NSAIDs in the long term ${ }^{2}$ because the use of these drugs has been associated with an increased risk of intestinal disease flares ${ }^{4,35}$. COX-2 inhibitors are presented as an attractive alternative to NSAIDs in patients with $\mathrm{IBD}^{36,37}$. Other treatments such as mesalamine are not effective in the treatment of arthropathies associated with IBD and therefore anti-TNF should be used early?

Recommendations for the treatment of peripheral arthropathy include treatment of intestinal inflammation, short-term NSAIDs, and local infiltration with corticosteroids. Other alternatives in these patients are shortterm oral corticosteroids, sulfasalazine, methotrexate, or anti-TNF².

\section{Oral and cutaneous manifestations}

These manifestations are diverse and are divided into several groups: manifestations with the same histology as IBD (metastatic CD), reactive manifestations (erythema nodosum, pyoderma gangrenosum, and Sweet's syndrome), dermatoses associated with IBD (psoriasis), and manifestations secondary to pharmacological treatment, especially secondary to anti-TNF (Table 2 and Fig. 2) ${ }^{38}$. The most frequent orocutaneous manifestations are described below.

\section{Erythema nodosum}

The diagnosis is based on the typical clinical presentation as raised subcutaneous nodules, red or purple,

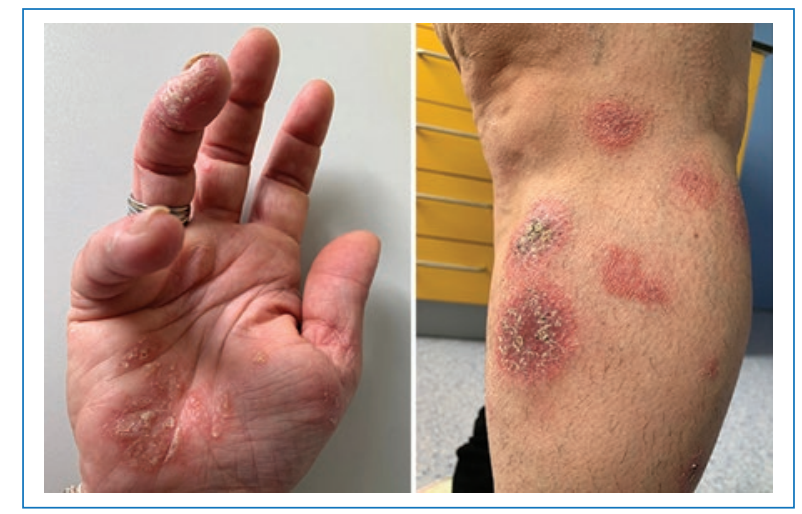

Figure 2. Anti-TNF-induced psoriasiform dermatitis.

hot, painful and between 1 and $5 \mathrm{~cm}$. Nodules are usually located in the pretibial region ${ }^{2}$. The appearance of this lesion is mostly accompanied by intestinal disease activity, although the severity of the erythema nodosum does not correlate with the severity of the intestinal symptoms $^{39}$. When the presentation or location is not typical, a skin biopsy can be used. Metastatic CD, Behçet's disease, sarcoidosis, and skin infections should be considered in the differential diagnosis of $\mathrm{EN}^{40}$.

Erythema nodosum usually resolves with an effective treatment for the bowel disease.

\section{Pyoderma gangrenosum}

It occurs more frequently in UC than in CD. Pyoderma gangrenosum presents as one or multiple erythematous and painful papules or pustules that progress to deep ulcers with sterile exudate. The most frequently affected areas are the pimples and the peristomal skin. The clinical course is not always parallel to that of intestinal activity. The diagnosis is made by the clinical presentation and by the exclusion of other diseases. Biopsy can help to exclude other cutaneous diseases even though findings are non-specific. Skin biopsy may not be needed if the clinical presentation is typical and if there is a good response to treatment ${ }^{40}$. Treatment will include systemic corticosteroids and, in refractory cases, infliximab, adalimumab, or oral or topical calcineurin inhibitors ${ }^{2}$.

\section{Sweet syndrome}

Also known as acute febrile neutrophilic dermatosis, it is a rare cutaneous EIM. It presents as soft, erythematous papules or nodules. Sweet syndrome usually runs in parallel with the intestinal activity ${ }^{40}$. Treatment is based on systemic corticosteroids and in refractory cases, immunosuppressants ${ }^{2}$. 
Table 2. Anti-TNF-induced skin lesions

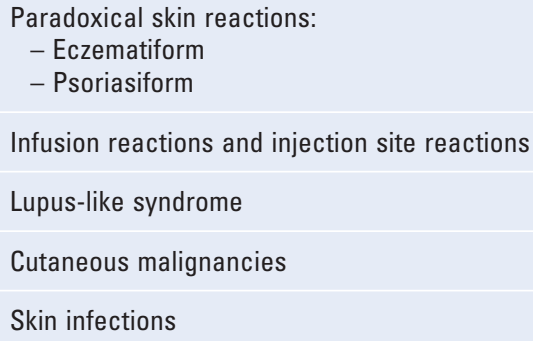

\section{Oral lesions}

Oral involvement is common in patients with IBD, and some of the following lesions may occur aphthous stomatitis, periodontitis, and vegetative peristomatitis. Oral lesions parallel intestinal disease activity, so the treatment will be that of the latter ${ }^{40}$.

\section{Ophthalmological manifestations}

After the joints and the skin, the eye is the organ most affected by EIMs. Within this kind of EIMs, episcleritis (Fig. 3) and anterior uveitis are the most frequent. Other ophthalmological manifestations such as scleritis (Fig. 4) or intermediate or posterior uveitis are much more infrequent. Episcleritis refers to the appearance of erythema in the sclera and conjunctiva and is usually painless. Anterior uveitis is less frequent and in patients with IBD, it is more frequently bilateral and of insidious onset. Associated symptoms are eye pain, blurred vision, photophobia, and headache. Normally, episcleritis parallels intestinal activity while uveitis runs and independent course ${ }^{3}$.

Treatment of episcleritis is the same as for IBD; topical or oral NSAIDs and topical corticosteroids are used to manage symptoms. On the other hand, uveitis deserves prompt referral to an ophthalmologist; topical corticosteroids may be used initially to reduce inflammation and topical cycloplegics to avoid spasms of the ciliary body and pupil and prevent the formation of synechiae. In refractory cases, systemic corticosteroids, immunosuppressants, or biologicals agents may be required ${ }^{2,41}$.

\section{Hepatobiliary manifestations}

The most common liver EIM is PSC. Even so, it should be noted that a percentage of patients with IBD may have an altered liver profile and first, hepatotoxicity (DILI) and other causes of liver disease independent of IBD must be ruled out.

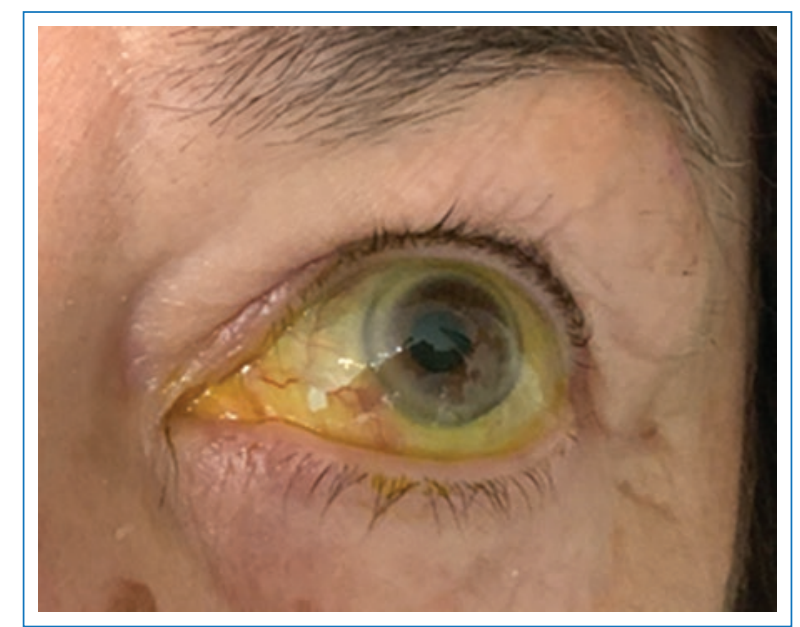

Figure 3. Epiescleritis.

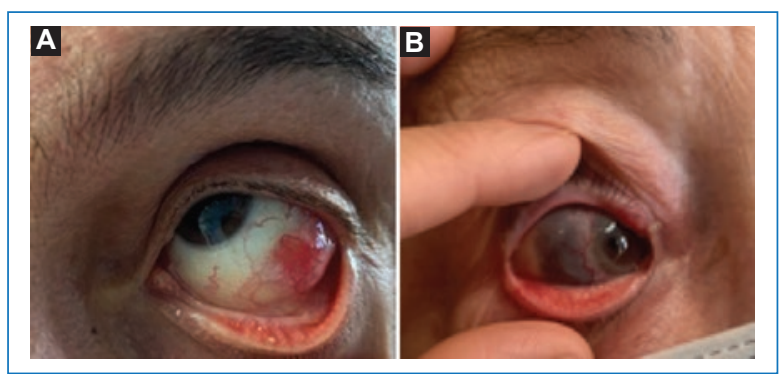

Figure 4. A: active scleritis. B: inactive scleritis with sclera thinning.

\section{Primary sclerosing cholangitis}

The diagnosis of PSC is made in a patient with cholestasis (elevated alkaline phosphatase is the most frequent laboratory finding) and typical features in magnetic resonance cholangiography (MRC), including multifocal intra- and extra-hepatic biliary strictures, once the causes of secondary sclerosing cholangitis have been ruled out (Table 3). In patients with normal MRC, a liver biopsy should be used to diagnose the smallduct PSC variant, which is characterized by normal appearance of the biliary tract in MRC $\mathrm{MR}^{2,42}$.

Between 24 and $96 \%$ of patients with PSC are positive for atypical perinuclear antineutrophil cytoplasmic autoantibody (p-ANCA $)^{42}$. However, this marker is not specific to this disease nor do they have prognostic significance. In those patients with elevated antinuclear antibodies, anti-smooth muscle antibodies, or increased immunoglobulin G, we should suspect the existence of autoimmune hepatitis (AlH) and perform a liver biopsy. If IgG4 concentrations are high (more than 4 times the normal value) or the lgG4/IgG1 ratio is increased, we should suspect sclerosing cholangitis associated with $\lg G 4^{2,43}$. 
As previously described, MRC is the diagnostic test of choice. The typical image shows short multifocal strictures in the intra- and extra-hepatic bile ducts. Endoscopic retrograde cholangiopancreatography (ERCP) is reserved for the treatment of strictures and to rule out cholangiocarcinoma (taking biopsies and brushing). In the case of ERCP, it is recommended to use prophylactic antibiotic therapy in all cases ${ }^{43}$.

The diagnosis of PSC in a patient with IBD significantly worsens the prognosis. Complications associated with PSC are cholestasis, cholangitis, cholelithiasis, cholangiocarcinoma, colorectal cancer, osteoporosis, vitamin deficiency, and steatorrhea ${ }^{2}$.

Regarding treatment, there are no drugs that have proven to prolong transplant-free survival in these patients. Even so, ursodeoxycholic acid is routinely used at intermediate doses of $15-20 \mathrm{mg} / \mathrm{kg} /$ day. This drug has been shown to improve cholestasis but not survival. High doses should be avoided. Corticosteroids and immunosuppressants can be used in patients with evidence of autoimmune hepatitis. The indications for liver transplantation in patients with PSC are decompensated liver disease, intractable pruritus, and recurrent bacterial cholangitis. Transplantation could also be considered in patients diagnosed of biliary dysplasia ${ }^{2,43}$.

The follow-up of patients with PSC will be done with annual (or biennial) colonoscopy for colorectal cancer screening ${ }^{43}$. There are no established recommendations for the early diagnosis of biliary neoplasms. In general, annual abdominal ultrasound is recommended to assess gallbladder lesions and perform additional tests (MRC, ERCP, and CT) if cholangiocarcinoma is suspected ${ }^{2}$.

\section{Cardiovascular manifestations}

The incidence of cardiovascular events in patients with IBD is low but higher than in general population. This risk must be taken into account given the morbidity and mortality associated with these events. Even so, an increase in cardiovascular mortality has not been demonstrated in patients with IBD compared to the general population ${ }^{44}$.

The cardiovascular manifestations are pericarditis, myocarditis, venous and arterial thromboembolism, arrhythmias, atrioventricular block, heart failure, endocarditis, valvular heart disease, and Takayasu arteritis. Pericarditis is the most common disease, representing $70 \%$ of cardiovascular manifestations ${ }^{44}$. IBD patients have 1.2 times higher risk of myocardial infarctions than general population, 1.2 times the risk of stroke, and 3.5 times the risk of mesenteric ischemia ${ }^{45,46}$.
Table 3. Causes of secondary sclerosing cholangitis

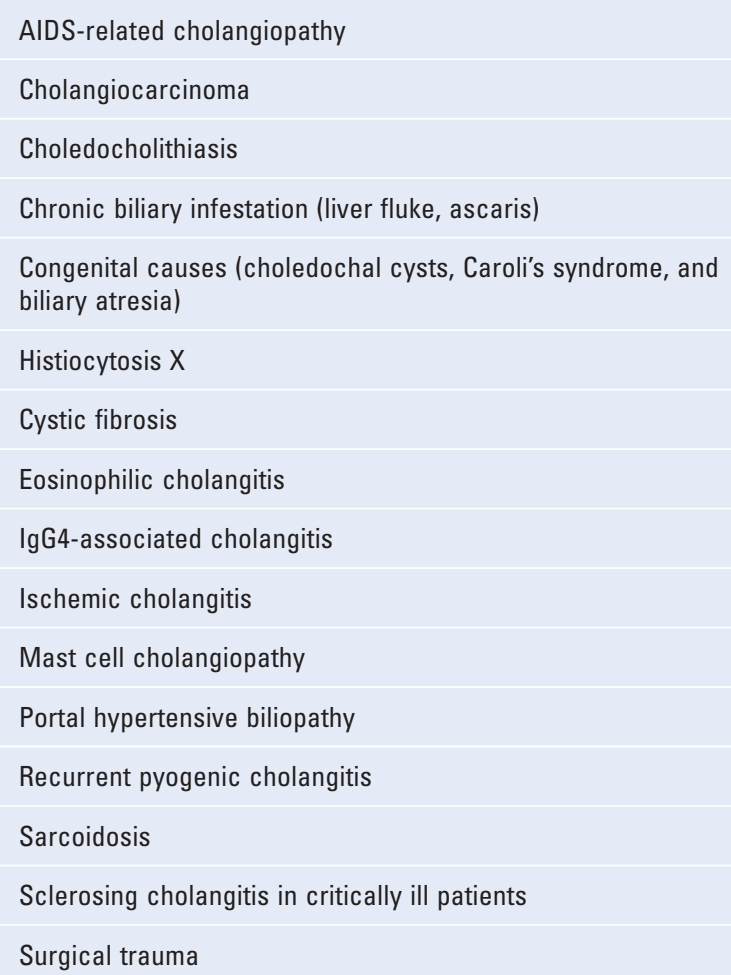

Venous thromboembolism (VTE) is approximately 3 times more common in patients with IBD than in the general population ${ }^{47}$. It mainly affects the lower limbs and the pulmonary circulation. Risk factors are an increased Charlson Comorbidity Index, advanced age, immobility, smoking, anemia, corticosteroids, central venous catheters, oral contraceptives, hospitalization, and recent surgery. There is an increased risk of developing VTE during periods of IBD flare compared to periods of remission ${ }^{3}$. Treatment is the same as in the general population. To prevent VTE events, the European consensus document of $\mathrm{ECCO}^{2}$ recommends antithrombotic prophylaxis for all patients with IBD admitted to a hospital and recommends to consider maintaining prophylaxis at discharge, after recent surgery and in outpatients with inflammatory activity ${ }^{2,44}$.

A key aspect of preventing cardiovascular events in patients with IBD is the prevention of flares and maintenance of remission because systemic inflammation is associated with the development of arteriosclerosis and coronary heart disease ${ }^{44}$.

\section{Pulmonary manifestations}

In those patients with IBD who present pulmonary symptoms in the first place, infections and 
drug-induced lung injury, especially due to 5-aminosalycilic acid (5-ASA) and methotrexate, must be ruled out. Salicylates are associated with different types of interstitial lung disease. Methotrexate can cause hypersensitivity pneumonitis or pulmonary fibrosis. Lung damage has also been described with the use of azathioprine, 6-mercaptopurine, and anti-TNF. Treatment essentially lies in the suspension of the drug involved ${ }^{2,48}$.

The pulmonary EIMs are rare. However, abnormalities found incidentally in respiratory function tests or chest imaging tests are relatively frequent. The EIMs of IBD usually affect the airway and can cause symptoms in any section of the respiratory tract from the glottis to small airways, most commonly affecting large airways. Lung interstitial damage can also occur, with organized pneumonia being the most common form. These manifestations usually respond adequately to treatment with inhaled or systemic corticosteroids. In refractory cases, immunosuppressants or biologicals may be used ${ }^{2,48}$

\section{Renal and urological manifestations}

Renal failure in patients with IBD may be due to drug toxicity or be an EIM of the disease, but this is difficult to differentiate. An association of IBD with the development of secondary amyloidosis, tubulointerstitial nephritis, and glomerulonephritis, especially lgA nephropathy, has been described. The risk of tubulointerstitial nephritis must be taken into account in patients treated with 5-ASA. Cyclosporine can cause acute and chronic kidney failure ${ }^{2,3,49}$.

Patients with IBD, especially those with $C D$, have more commonly uric acid or calcium oxalate nephrolithiasis ${ }^{2}$. An important proportion of IBD patients with nephrolithiasis has undergone surgery. Low urine $\mathrm{pH}$ and low urine volumes are risk factors associated with the development of uric acid stones. Patients with IBD have also a higher risk of calcium oxalate nephrolithiasis as a result of an increased intestinal oxalate absorption ${ }^{50}$.

\section{Osteopenia and osteoporosis}

Osteopenia and osteoporosis are common in patients with IBD. The prevalence of osteoporosis varies between $4 \%$ and $9 \%$. The diagnosis of osteoporosis is made when T-score is lower than -2.5 on a radiographic bone densitometry, whereas osteopenia is defined as a T-score between -1 and $-2.5^{2,51}$.

$\mathrm{CD}$, a low body mass index, and low weight are risk factors associated with low bone mineral density (BMD) or osteoporosis ${ }^{51}$. Some measures such as wear bearing exercise, smoking cessation, and an adequate intake of calcium in the diet $(1 \mathrm{~g} /$ day) prevent the loss of BMD. The European consensus document of $\mathrm{ECCO}^{2}$ recommends systematically calcium and Vitamin $D$ in patients receiving systemic corticosteroids. Control of intestinal inflammatory activity is necessary to reduce bone loss. Bisphosphonate treatment has been shown to reduce the risk of vertebral fractures in patients with IBD. Even so, this treatment cannot be widely recommended in young patients and premenopausal women ${ }^{2,52}$.

\section{Conclusion}

IBD is a systemic inflammatory disease that affects multiple systems other than the intestine. The symptoms, treatment, and prognosis of different EIMs are varied. Its pathogenesis is still unclear and its deeper knowledge would help us to better treat them. Some EIMs run an independent course from the IBD and can affect severely patient's quality of life, so the treating IBD physician should be aware to detect and control this EIM in due course.

\section{Funding}

No funding was needed for the writing of this manuscript.

\section{Conflicts of interest}

Ignacio Marín-Jiménez has served as a consultant, advisory member, speaker, or has received research funding from MSD, Abbvie, Takeda, Tillots, Ferring, Falk-Pharma, Faes Farma, UCB Pharma, Otsuka Pharmaceutical, Shire, Gebro Pharma, Pfizer, Biogen, Sandoz, Fresenius, and Chiesi.

C. R-B and LM declare no conflicts of interest.

\section{Ethical disclosures}

Protection of human and animal subjects. The authors declare that no experiments were performed on humans or animals for this study.

Confidentiality of data. The authors declare that no patient data appear in this article.

Right to privacy and informed consent. The authors declare that no patient data appear in this article.

\section{References}

1. Vavricka SR, Rogler G, Gantenbein C, Spoerri M, Vavricka MP Navarini AA, et al. Chronological order of appearance of extraintestinal manifestations relative to the time of IBD diagnosis in the swiss inflammatory bowel disease cohort. Inflamm Bowel Dis. 2015;21:1794-800. 
2. Harbord M, Annese V, Vavricka SR, Allez M, de Acosta MB, Boberg KM, et al. The first european evidence-based consensus on extra-intestinal manifestations in inflammatory bowel disease. J Crohns Colitis. 2016;10:239-54

3. Garber A, Regueiro M. Extraintestinal manifestations of inflammatory bowel disease: epidemiology, etiopathogenesis, and management. Curr Gastroenterol Rep. 2019;21:31.

4. Rogler G, Singh A, Kavanaugh A, Rubin DT. Extraintestinal manifestations of inflammatory bowel disease: current concepts, treatment, and implications for disease management. Gastroenterology. 2021;161:1118-32.

5. Danese S, Semeraro S, Papa A, Roberto I, Scaldaferri F, Fedeli G, et al. Extraintestinal manifestations in inflammatory bowel disease. World $J$ Gastroenterol. 2005;11:7227-36

6. Bernstein CN, Blanchard JF, Rawsthorne P, Yu N. The prevalence of extraintestinal diseases in inflammatory bowel disease: a population-based study. Am J Gastroenterol. 2001;96:1116-22.

7. Ricart E, Panaccione R, Loftus EV, Tremaine WJ, Harmsen WS, Zinsmeister AR, et al. Autoimmune disorders and extraintestinal manifestations in first-degree familial and sporadic inflammatory bowel disease: a case-control study. Inflamm Bowel Dis. 2004;10:207-14.

8. Mendoza JL, Lana R, Taxonera C, Alba C, Izquierdo S, Díaz-Rubio M. Manifestaciones extraintestinales en la enfermedad inflamatoria intestinal: diferencias entre la enfermedad de crohn y la colitis ulcerosa. Med Clin (Barc). 2005;125:297-300.

9. Rankin GB, Watts HD, Melnyk CS, Kelley ML. National cooperative crohn's disease study: extraintestinal manifestations and perianal complications. Gastroenterology. 1979;77:914-20

10. Su CG, Judge TA, Lichtenstein GR. Extraintestinal manifestations of inflammatory bowel disease. Gastroenterol Clin North Am. 2002;31:307-27.

11. Greenstein AJ, Janowitz HD, Sachar DB. Greenstein. Medicine. 1976;55:401-12.

12. Farmer RG, Hawk WA, Turnbull RB. Clinical patterns in crohn's disease: a statistical study of 615 cases. Gastroenterology. 1975;68:627-35.

13. Veloso F, Carvalho J, Magro F. Immune-related systemic manifestations of inflammatory bowel disease. A prospective study of 792 patients. J Clin Gastroenterol. 1996;23:29-34.

14. Vind I, Riis L, Jess T, Knudsen E, Pedersen N, Elkjær M, et al. Increasing incidences of inflammatory bowel disease and decreasing surgery rates in Copenhagen city and county, 2003-2005: a population-based study from the Danish Crohn colitis database. Am J Gastroenterol. 2006:101:1274-82

15. Vavricka SR, Brun L, Ballabeni P, Pittet V, Prinz Vavricka BM, Zeitz J, et al. Frequency and risk factors for extraintestinal manifestations in the swiss inflammatory bowel disease cohort. Am J Gastroenterol. 2011:106:110-9.

16. Hedin CR, Vavricka SR, Stagg AJ, Schoepfer A, Raine T, Puig L, et al. The pathogenesis of extraintestinal manifestations: Implications for IBD research, diagnosis, and therapy. J Crohns Colitis 2019;13:541-54.

17. Grant AJ, Lalor PF, Hübscher SG, Briskin M, Adams DH. MAdCAM-1 expressed in chronic inflammatory liver disease supports mucosal lymphocyte adhesion to hepatic endothelium (MAdCAM-1 in chronic inflammatory liver disease). Hepatology. 2001:33:1065-72.

18. Eksteen B, Grant AJ, Miles A, Curbishley SM, Lalor PF, Hübscher SG et al. Hepatic endothelial CCL25 mediates the recruitment of CCR9+ gut-homing lymphocytes to the liver in primary sclerosing cholangitis. J Exp Med. 2004;200:1511-7.

19. Salmi M, Andrew DP, Butcher EC, Jalkanen S. Dual binding capacity of mucosal immunoblasts to mucosal and synovial endothellum in humans: dissection of the molecular mechanisms. J Exp Med. 1995;181:137-49.

20. Geng X, Biancone L, Dai HH, Lin JJ, Yoshizaki N, Dasgupta A, et al. Tropomyosin isoforms in intestinal mucosa: production of autoantibodies to tropomyosin isoforms in ulcerative colitis. Gastroenterology. 1998; $114: 912-22$

21. Das KM, Vecchi M, Sakamaki S. A shared and unique epitope(s) on human colon, skin, and biliary epithelium detected by a monoclonal antibody. Gastroenterology. 1990;98:464-9.

22. Bhagat S, Das KM. A shared and unique peptide in the human colon, eye, and joint detected by a monoclonal antibody. Gastroenterology. 1994;107:103-8.

23. Kummen M, Holm K, Anmarkrud JA, Nygård S, Vesterhus M, Høivik ML, et al. The gut microbial profile in patients with primary sclerosing cholangitis is distinct from patients with ulcerative colitis without biliary disease and healthy controls. Gut. 2017;66:611-9.

24. Torres J, Bao X, Goel A, Colombel JF, Pekow J, Jabri B, et al. The features of mucosa-associated microbiota in primary sclerosing cholangitis. Aliment Pharmacol Ther. 2016;43:790-801.

25. Park JH, Peyrin-Biroulet L, Eisenhut M, Shin JI. IBD immunopathogenesis: a comprehensive review of inflammatory molecules. Autoimmun Rev. 2017:16:416-26

26. Condliffe AM, Kitchen E, Chilvers ER. Neutrophil priming: pathophysiological consequences and underlying mechanisms. Clin Sci. 1998:94:461-71.

27. Griseri T, McKenzie BS, Schiering C, Powrie F. Dysregulated hematopoietic stem and progenitor cell activity promotes interleukin-23-driven chronic intestinal inflammation. Immunity. 2012;37:1116-29.
28. Crost $E H$, Tailford LE, Monestier M, Swarbreck D, Henrissat $B$, Crossman LC, et al. The mucin-degradation strategy of Ruminococcus gnavus: the importance of intramolecular trans-sialidases. Gut Microbes. 2016;7:302-12.

29. Hedin CR, van der Gast CJ, Stagg AJ, Lindsay JO, Whelan K. The gut microbiota of siblings offers insights into microbial pathogenesis of inflammatory bowel disease. Gut Microbes. 2017:8:1-7.

30. Purchiaroni F, Tortora A, Gabrielli M, Bertucci F, Gigante G, laniro G, et al. The role of intestinal microbiota and the immune system. Eur Rev Med Pharmacol Sci. 2013;17:323-33.

31. Salvarani C, Fries W. Clinical features and epidemiology of spondyloarthritides associated with inflammatory bowel disease. World J Gastroenterol. 2009;15:2449-55.

32. González-Lama Y, Sans J, Bastida G, Campos J, Ferreiro R, Joven B et al. Recomendaciones del grupo español de trabajo en enfermedad de crohn y colitis ulcerosa (GETECCU) sobre el cribado y tratamiento de la infección tuberculosa en pacientes con enfermedad inflamatoria intestinal. Gastroenterol Hepatol. 2021;44:51-66.

33. Steer S, Jones H, Hibbert J, Kondeatis E, Vaughan R, Sanderson J, et al. Low back pain, sacroiliitis, and the relationship with HLA-B27 in Crohn's disease. J Rheumatol. 2003:30:518-22.

34. Orchard TR, Wordsworth BP, Jewell DP. Peripheral arthropathies in inflammatory bowel disease: their articular distribution and natural history. Gut. 1998;42:387-91.

35. Felder JB, Korelitz BI, Rajapakse R, Schwarz S, Horatagis AP, Gleim G. Effects of nonsteroidal antiinflammatory drugs on inflammatory bowe disease: a case-control study. Am J Gastroenterol. 2000:95:1949-54.

36. Sandborn WJ, Stenson WF, Brynskov J, Lorenz RG, Steidle GM Robbins JL, et al. Safety of celecoxib in patients with ulcerative colitis in remission: a randomized, placebo-controlled, pilot study. Clin Gastroenterol Hepatol. 2006;4:203-11.

37. El Miedany Y, Youssef S, Ahmed I, El Gaafary M. The gastrointestinal safety and effect on disease activity of etoricoxib, a selective Cox-2 inhibitor in inflammatory bowel diseases. Am J Gastroenterol. 2006;101:311-7.

38. Lambert JLW, De Schepper S, Speeckaert R. Cutaneous manifestations in biological-treated inflammatory bowel disease patients: a narrative review. J Clin Med. 2021;10:1040.

39. Apgar J. Newer aspects of inflammatory bowel disease and its cutaneous manifestations: a selective review. Semin Dermatol. 1991;10:138-47.

40. Greuter T, Navarini A, Vavricka SR. Skin manifestations of inflammatory bowel disease. Clin Rev Allergy Immunol. 2017;53:413-27.

41. Troncoso LL, Biancardi AL, de Moraes HV, Zaltman C. Ophtalmic manifestations in patients with inflammatory bowel disease: a review. World J Gastroenterol. 2017;23:5836-48.

42. European Association for the Study of the Liver. EASL clinical practice guidelines: management of cholestatic liver diseases. J Hepatol. 2009:51:237-67.

43. Dyson JK, Beuers U, Jones DE, Lohse AW, Hudson M. Primary sclerosing cholangitis. Lancet. 2018;391:2547-59.

44. Bunu DM, Timofte CE, Ciocoiu M, Floria M, Tarniceriu CC, Barboi OB, et al. Cardiovascular manifestations of inflammatory bowel disease: pathogenesis, diagnosis, and preventive strategies. Gastroenterol Res Pract. 2019;2019:3012509.

45. Fumery M, Xiaocang C, Dauchet L, Gower-Rousseau C, Peyrin-Biroulet L, Colombel JF. Thromboembolic events and cardiovascular mortality in inflammatory bowel diseases: a meta-analysis of observational studies. J Crohns Colitis. 2014;8:469-79.

46. Singh S, Singh H, Loftus EV, Pardi DS. Risk of cerebrovascular accidents and ischemic heart disease in patients with inflammatory bowel disease: a systematic review and meta-analysis. Clin Gastroenterol Hepatol. 2014;12:382-93.e1.

47. Bernstein C, Blanchard J, Houston D, Wajda A. The incidence of deep venous thrombosis and pulmonary embolism among patients with inflammatory bowel disease: a population-based cohort study. Thromb Haemost. 2001;85:430-4.

48. Massart A, Hunt DP. Pulmonary manifestations of inflammatory bowel disease. Am J Med. 2020;133:39-43.

49. Ambruzs JM, Larsen CP. Renal manifestations of inflammatory bowel disease. Rheum Dis Clin North Am. 2018;44:699-714.

50. Oikonomou K, Kapsoritakis A, Eleftheriadis T, Stefanidis I, Potamianos S Renal manifestations and complications of inflammatory bowel disease. Inflamm Bowel Dis. 2011;17:1034-45.

51. Kärnsund S, Lo B, Bendtsen F, Holm J, Burisch J. Systematic review of the prevalence and development of osteoporosis or low bone mineral density and its risk factors in patients with inflammatory bowel disease. World J Gastroenterol. 2020;26:5362-74.

52. Melek J, Sakuraba A. Efficacy and safety of medical therapy for low bone mineral density in patients with inflammatory bowel disease: a meta-analysis and systematic review. Clin Gastroenterol Hepatol. 2014;12:32-44.e5.

53. Greuter T, Vavricka SR. Extraintestinal manifestations in inflammatory bowel disease-epidemiology, genetics, and pathogenesis. Expert Rev Gastroenterol Hepatol. 2019;13:307-17. 